\title{
Electron hybridization and anharmonic thermal vibration effect on structure transition of $\mathrm{SrTiO}_{3}$ at high-pressure and low-temperature.
}

\author{
Takamitsu Yamanaka*1. ${ }^{\text {. Muhtar Ahart }}{ }^{1}$, Ho-kwang Mao ${ }^{1}$, Russell J. Hemley ${ }^{1}$, \\ and Takeyuki Suzuki ${ }^{2}$
}

\author{
${ }^{1}$ Carnegie Institution of Washington Geophysical Laboratory, \\ 5251 Broad Branch Rd N.W. Washington DC 20015 \\ ${ }^{2}$ Comprehensive Analysis Center, Institute of Science and Industrial Research, \\ Osaka University $\quad$ 8-1 Mihogadake Ibaraki Osaka 567-0047 \\ * Corresponding author \\ E-mail address: t.yamanaka@cap.ocn.ne.jp
}

\section{Abstract}

We execute electron density analysis of $\mathrm{SrTiO}_{3}$ at low-temperatures up $80 \mathrm{~K}$ and high-pressures up to 11.88 GPa using X-ray single-crystal diffraction experiment and ab initio quantum chemical molecular orbital (MO) calculation. The lattice constants of $a$ and $c$ of tetragonal phase are noticeably decreased with pressure above $\mathrm{P}_{\mathrm{c}}$ in comparison with those at low temperature below $\mathrm{T}_{\mathrm{c}}$. The lattice constant ratio of $c / a<1$ of the tetragonal phase becomes more extreme with increasing pressure. On the other hand, the ratio below $T_{c}$ is $c / a>1$ and increases with lowering temperature.

Difference Fourier (D-F) synthesis experimentally proves the residual electron densities $\Delta \rho(\mathrm{xyz})$ are associated with two different effects: electron hybridization bonding electron and anharmonic thermal vibration atoms. The $d-p-\pi$ hybridization between $\operatorname{Ti}(3 d)$ and $\mathrm{O}(2 p)$ orbitals is confirmed in the residual electron density, which is deformed from the ideal spherical density conducted by the atomic scattering factor $f i$ using Hartree-Fock (HF) approximation. MO calculation also reveals the electron hybridization. Anharmonic thermal vibration of atoms yields a large effect to the structure transition. Mulliken charges analysis of MO calculation indicates much smaller charges than their formal ionic charges. Their ionicity increases from cubic to tetragonal above $P_{c}$ and below $\mathrm{T}_{\mathrm{c}}$. 


\section{$30 \quad$ Keywords}

31 Structure transition at high-pressure and low-temperature; Anharmonic thermal vibration:

$32 d-p-\pi$ hybridization electron density; Molecular orbital calculation

33 


\section{Introduction}

Structure changes of ferroelectric crystals under extreme conditions are often induced from the phonon-electron interaction. An antiferro-distortive phase transition of $\mathrm{SrTiO}_{3}$ (STO) at $105 \mathrm{~K}$ was reported [1][2]. At low-temperatures below around $37 \mathrm{~K}$, soft phonon anomaly and quantum fluctuation for the transition were suggested by electron paramagnetic resonance and dielectric measurement [3] [4][5][6]. A super conducting state was confirmed [7]. Optical investigations by Raman and infrared spectroscopy have been also executed for STO at low temperature [8][9]. First principles calculation proposed the transition from ferroelectric to antiferro-distortion in tetrahedral phase [10][11][12][13]. Ab initio molecular orbital calculation (MO) has been intensively undertaken to elucidate the dynamical structure change [14][15]. Evidence for a phase transition of STO on (001) surface at low-temperature was proved by RHEED [16].

A cubic-tetragonal phase boundary is proposed by the Landau theory of phase transitions with the coupling effect between the order parameter and the volume spontaneous strain [8]. The phase transition mechanism is characterized by the distortion and tilt of the $\mathrm{TiO}_{6}$ octahedra in the tetragonal phase with nonlinear couplings between the structural order parameter and the volume strain as a function of the applied pressure [17][18][19].

Experimental determination of the charge density in cubic STO has been reported by studies employing synchrotron [20], laboratory X-rays [21] and $\gamma$-ray diffraction at room temperature [22].

The topological analysis of the electron density of STO was discussed based on the X-ray diffraction study at $145 \mathrm{~K}$ and theoretical calculation using the density functional formulae [23]. The analysis of the kinetic and potential energy density, derived from the electron density, reveals the stabilizing crystal-forming role of the $\mathrm{O}$ atoms in STO. The experimental electron density and the kinetic energy densities are observed [24]. Calculations of electron densities are carried out from X-ray diffraction data [25].

We aim to elucidate static atomic positional disorder using X-ray single-crystal diffraction experiment of STO at low-temperatures and high-pressures. Difference Fourier synthesis discloses the residual electron density distribution in consideration of bonding electrons and anharmonic thermal vibration. Quantum chemical MO calculation is also undertaken to confirm the effective charge of atoms at the transition under low-temperature and high-pressure conditions.

\section{Experiment}

Sample preparation of single crystal STO is described in supplement. Sample preparation gives large 
effects on structure transition and Curie point.

Single crystal diffraction study was undertaken using four-circle diffractometer of RIGAK AFC7 with point detector of scintillation counter on $18 \mathrm{~kW}$ rotated anode X-ray generator. The highly collimated beam is applied for diffraction experiments under extreme conditions. Precise diffraction intensity measurement at low-temperatures down to $80 \mathrm{~K}$ was undertaken. Diffraction studies at high pressures up to11.88 GPa were made using diamond anvil cell [DAC] designed for single crystal diffraction study [26]. Pressure is measured by the ruby fluorescence system [27].

The structure factor $F_{o b s}$ is expressed by

$$
F_{c a l}(\vec{h})=K \sum_{j} a_{j} f_{j}(\vec{h}) \sum T_{j s}(\vec{h}) \exp \left\{2 \pi i\left(h x_{j s}+k y_{j s}+l z_{j s}\right)\right\}
$$

Atomic scattering factor $f_{j}$ of $j$ atom was provided by Hartree-Fock approximation. Anomalous dispersion parameters were taken into account in $f=f_{o}+\Delta f^{\prime}+i \Delta f^{\prime \prime}$. The least-squares refinement is conducted by minimization of $\Delta: \quad \Delta=\sum_{\vec{h}} w(\vec{h})\left\{\left|F_{\text {obs }}(\vec{h})\right|-\left|F_{c a l}(\vec{h})\right|\right\}^{2}$

\section{Result and discussion}

The lattice constants were determined by least-square calculation based on the peak refinement of 25 reflections of the single-crystal diffraction. The lattice constant changes as a function of temperature and pressure are presented in Table A.1 and A.2 in appendices. The transition pressure $\left(\mathrm{P}_{c}\right)$ from cubic to tetragonal phase is at about $7 \mathrm{GPa}$ and in the low-temperature experiments the transition temperature $\left(\mathrm{T}_{\mathrm{c}}\right)$ is about $100 \mathrm{~K}$. These $\mathrm{P}_{\mathrm{c}}$ and $\mathrm{T}_{\mathrm{c}}$ are in a good agreement with previously reported data [7].

In order to clarify the pressure and temperature dependence of the lattice constants, two compression curves are presented in Fig. 1. The compression of $a$ and $c$ of the tetragonal phase are noticeably decreased at high-pressures in comparison with those at low-temperature. The ratio of $c / a$ of the tetragonal phase in high-pressure with $c / a<1$ becomes more extreme with increasing pressure above $\mathrm{P}_{\mathrm{c}}$. On the other hand, the ratio at low temperature below $T_{c}$ is $c / a>1$ and increases with lowering temperature. The observed lattice constants and atomic positions of two phases at high-pressure and low-temperature are listed in Table A.1 and A.2 in appendices respectively. The tetragonal structures are formed by rotation of $\mathrm{TiO}_{6}$ octahedron around the $c$ axis due to the displacement of oxygen atoms.

Two Ti-O1 distances enormously decrease in the tetragonal structure at both low-temperature and high-pressure. On the other hand, four Ti-O2 increases with pressure. The rotation of four O2 in the (001) plane 
with keeping the four-fold rotation axis is more extended with increasing pressure. The bond distance ratio of Ti-O2/Ti-O1 of the tetragonal phase induces the distortion of octahedron of $\mathrm{TiO}_{6}$. The bond distance ratio of the tetragonal phase at high-pressure is much larger than at low-temperature, as shown in Table 1. Since the rotation induced by the compression is related to $c / a<1$ in the tetragonal phase, the more rotation promotes the distinct changes. The bond distance changes with pressure and temperature are presented in Fig. A.1 in appendices. Every four $\mathrm{Sr}-\mathrm{O} 1$ and $\mathrm{Sr}-\mathrm{O} 2$ distances in the tetragonal phase remarkably decreases with pressure above $\mathrm{P}_{c}$, although Sr has twelve coordinations with $\mathrm{O}$ in the cubic phase.

Pressure effect to the structure is much more remarkable than thermal effect. Average bond $<\mathrm{Sr}-\mathrm{O}>$ is much more compressed than $\langle$ Ti-O $>$ under the both conditions. The measurable tolerant factor of $t=\frac{<S r-O>}{\sqrt{2}<T i-O>}$ using the average bond lengths in Table 1 decreases with compression and cooling. The factor proves a more deformation of tetragonal lattice with these conditions.

\section{Electron density distribution}

Difference Fourier synthesis (D-F) of $\left\{\left|F_{o b s}(h k l)\right|-\left|F_{c a}(h k l)\right|\right\}$ discloses the deformation electron density (ED) distribution. The residual ED $\Delta \rho(x y z)$ expresses the nonspherical deformation electron cloud by

$$
\Delta \rho(x y z)=\frac{1}{V} \sum_{h} \sum_{k} \sum_{l}\left\{\left|F_{o b s}(h k l)\right|-\left|F_{c a l}(h k l)\right|\right\} \exp \{-2 \pi i(h x+k y+l z)\}
$$

The atomic scattering factor $f i$ in the structure factor $F(h k l)$ applies Hartree-Fock approximation, which is composed of the spherical ED distribution model without any interaction from the nearest or second neighbor atoms. D-F maps based on the harmonic oscillator model show the deformation of ED around $\mathrm{Sr}$ and $\mathrm{Ti}$ atomic positions in the projection on to (001). Aspherical residual ED distributions are recognized around the Ti position at ambient conditions. The distributions show the non-spherical bonding electron and anisotropic anharmonic thermal vibration of atoms. The ED along the Ti-O bond is good agreement with the first principles calculations and molecular orbital calculations at ambient conditions [10][11][13][14][28].

Numbers of $3 d$-electrons of Ti are reduced with more ionization. Sr has an outer shell electron (valence electron) orbitals of only $5 s$ electron which is a spherical electron orbital. The D-F map at ambient conditions in 
118 Fig. 2 shows the $d-p-\pi$ hybridization between $t_{2 g}$ electron of $\operatorname{Ti}(3 d)$ and $p_{x}$ electron of $\mathrm{O}(2 p)$. The ED 119 distribution around Ti with increasing pressure is presented in Fig. A.2 in appendices. These maps prove the more localization of ED, resulting in more ionic character in the tetragonal phase. The localization is enhanced with increasing pressure above $P_{c}$. The $\pi$ bond character gradually disappears with successive pressure.

The same bonding nature of the $d-p-\pi$ bond in the octahedral site in the ferroelectric orthorhombic perovskite $\mathrm{KNbO}_{3}$ [29] and ferroelectric tetragonal perovskite $\mathrm{PbTiO}_{3}$ were found by maximum entropy method using the X-ray single crystal diffraction intensities. Ab initio model calculation [30] proves $\mathrm{O}$ valence electrons in $\mathrm{BaTiO}_{3}$ are strongly hybridized with Ti. The ED feature around Ti at ambient conditions accords with the ED calculations [22][31]. Sr valence shell electrons do not exhibit asphericity and deformation of the electron orbital, because of $5 s$ electron.

The difference of Ti-O and Sr-O bond distances in the tetragonal phase can be explained by Fig. A.1, in which the D-F maps of the tetragonal phase at $10.28 \mathrm{GPa}$ and $80 \mathrm{~K}$. The thermal vibration of the $\mathrm{O} 1$ atom interprets two positive peaks along the c axis around $\mathrm{O} 1$ in the map at $80 \mathrm{~K}$. Electron densities of both $\mathrm{Ti}$ and $\mathrm{Sr}$ are elongated to the direction of the $c$ axis. The $c / a>1$ of the tetragonal phase at low-temperature can be explained by the positional disorder in the direction of the $c$ axis. The D-F map at 10.28 GPa proves the residual ED is elongated to the $a$-direction. The two maps specify the difference in thermal dynamical vibration of atoms of the tetragonal phases under low-temperature and high-pressure conditions.

\section{Anharmonic thermal parameter}

The distortion of ferroelectric substance is disclosed by the thermal vibration of atoms, which is introduced as thermal parameters $T_{i j}(h k l)$ in the structure refinement. The parameters are indicators of the atomic positional disorder (lattice average) and dynamical disorder (time average) of the atomic vibration in the crystal structure. Harmonic oscillation of atom cannot make any shift of the atomic position, because the harmonic potential do not shift the minimum of the parabolic potential well. ED ascribed to the dynamical disorder can be discussed only by anisotropic anharmonic thermal vibration of atoms in consideration of higher-order tensors than the conventional second-rank thermal parameters.

From X-ray diffraction analysis, anharmonic thermal vibrations of all atoms are enhanced with thermal energy and cause the deformation of the lattice [26]. On the other hand, the localization of the valence electron around the cation position is more enhanced under higher-pressure. 
147 Anharmonic thermal analyses of titanate and niobate perovskite have been performed using synchrotron 148 radiation and hot neutrons [32][33]. The motions of the split atom model and the rotator model are obtained in 149 the present phase transition.

150 We adopted a multimodal distribution in the probability density function using cumulant expansion and 151 Gram-Charlier series expansion [34][35]. We take into account of fourth-rank in $T_{i j}(h k l)$ in eq. (1). Since the 152 factors above fifth-rank have a large correlation with each other, they are not reliable. Consequently they are not 153 applied in the calculation. The anharmonic equation of the cumulant and Gram-Charlier series expansions are 154 detailed in Table A.3 in appendices. They can be interpreted by the atomic positional disorder and atomic 155 vibration. Electron density in D-F maps of cubic STO based on harmonic model (left side) and anharmonic oscillation model (right site) are presented in Fig. 3.

Anharmonic model in the tetrahedral phase at low-temperatures reveals a large anisotropy in both $\mathrm{Sr}$ and $\mathrm{Ti}$. Anharmonic thermal vibrations of $\mathrm{O}$ (including $\mathrm{O} 1$ and $\mathrm{O} 2$ ) in both cubic and tetragonal phases are more obvious than $\mathrm{Sr}$ and $\mathrm{Ti} . \mathrm{O} 1$ and $\mathrm{O} 2$ show noticeable anisotropic anharmonic parameters below $\mathrm{T}_{\mathrm{c}}$, as shown Fig. 3. $\mathrm{O} 2$ has a variable positional parameter and more flexibility than $\mathrm{O} 1$.

\section{MO calculation and Mulliken charge}

The electronic structures, (band structure, density of state and ED distribution), have been examined with emphasis on the covalency effects of ferroelectrics. Structure studies of dynamical properties of perovskites have been published by LDA [36] and the local spin density approximation was reported by GGA calculations [37]. The electronic structure of a wide range of perovskites was examined using ab initio HF method [38]. A number of different approximations correlation functional including hybrid exchange techniques have been developed. Many methods are compared with previous quantum mechanical calculations [11][12][13][14].

In the present study, $a b$ initio calculation of the electronic structure is performed using the quantum chemical MO calculation program package of Gaussian-09 [38]. Structural models were based on the atomic coordinates experimentally determined by the precise structure analyses by single crystal X-ray diffraction study. The results of the calculation are visioalized by program GaussianView [39], which is the graphical interface available for Gaussian-09.

We applied HF and DFT for all calculations using program Gaussian-09 at different pressures and temperatures with the 3-21G and 6-31G basis set. We applied several structure models including $\mathrm{Sr}$, Ti and O 
together with $\mathrm{H}$ atoms to avoid the lone-paired electrons of oxygen. The selected structure models are the distribution of atoms perspective to $<100>,<110>$ and $<111>$ directions.

Surface of the highest occupied molecular orbitals surface at various pressures and temperature are presented in Fig. A.4 and Fig. A.5 in appendices. High-energy orbital surface indicates the $d-p$ - $\pi$ bond between Ti and O, which is shown in Fig. 4. The orbitals clearly show the $\pi$ bond in the figure. The calculated MO figures are in accordance with the D-F map observed by the residual ED distribution of the $d-p-\pi$ bond. No bonding orbital is found in MO around $\mathrm{Sr}$ atoms, because valence electron of $\mathrm{Sr}$ is $5 s$. Since MO calculation reveals the static electron orbits, it does not disclose the atomic vibration of atoms.

Mulliken charges indicate the effective charges for $\mathrm{Sr}, \mathrm{Ti}$ and $\mathrm{O}$ ions. The calculated charges are presented at variable temperatures and pressure in Table 2. The charges of $\mathrm{Ti}, \mathrm{Sr}$ and $\mathrm{O}$ are much smaller than their formal ionic charges. The covalent nature of the $\mathrm{Ti}-\mathrm{O}$ bond is proved, as already discussed from the residual ED in the D-F map. However, the charge of Sr remains a little closer to the formal charge. In the tetragonal structure at high-pressures above $\mathrm{P}_{c}, \mathrm{Ti}$ and $\mathrm{Sr}$ increase their ionic characters. Mulliken charges of Ti, $\mathrm{Sr}$ and $\mathrm{O}$ increase their ionicity with changing structure from cubic to tetragonal at high-pressures above $\mathrm{P}_{\mathrm{c}}$. The charge of $\mathrm{Ti}$ ion increases with increasing pressure and lowering temperature. It infers the perovskite structure is composed of the rigid octahedra of $\mathrm{TiO}_{6}$. But $\mathrm{Sr}$ atom seems to be placed in the open space. The ionicity is accelerated in the tetragonal phase. Trigger of the rotation may be induced by the approach of the compression toward the limit of the Ti-O bonds of antiferroelectric tetragonal phase.

\section{Conclusion}

Precise diffraction intensity measurement at extreme conditions can reveal ED in the D-F maps indicating the electron hybridization of $d-p-\pi$ bond of Ti and $\mathrm{O}$ electron around Ti. ED ascribed to the dynamical disorder can be discussed only by anisotropic anharmonic thermal vibration of atoms. The pressure dependence of ED is more localized and more ionized with elevating pressure.

The compressions of the lattice constants of $a$ and $c$ of tetragonal phase are noticeably decreased at pressures above $\mathrm{P}_{\mathrm{c}}$. The lattice distortion of $c / a<1$ of the tetragonal phase becomes more intensified with increasing pressure. On the other hand the ratio at low temperature below $T_{c}$ is $c / a>1$ and increases with lowering temperature. The difference in the lattice constants is induced by rotation of $\mathrm{O} 2$ atomic positions in two phases between high-pressure and low-temperature experiments. 
205 Observed Mulliken calculated by $A b$ initio $\mathrm{MO}$ charges of $\mathrm{Ti}$, $\mathrm{Sr}$ and $\mathrm{O}$ are much smaller charges than their 206 formal ionic charges. The increase in their charges indicates more ionic at the transition from cubic to tetragonal 207 above $\mathrm{P}_{\mathrm{c}}$ and below $\mathrm{T}_{\mathrm{c}}$, which is coincident with the experimental results of the $\mathrm{D}-\mathrm{F}$ observations.

\section{Acknowledgement}

210 We greatly appreciate Dr. A. Kyono in Graduate School of Life and Environmental Sciences, University of 211 Tsukuba for the sample preparation and express our thanks to Dr. Y. Nakamoto and Dr. M. Sakata, Center for 212 Science and Technology Under Extreme Conditions, Osaka University for their help for the sample 213 pressurization. This work was supported as a part of EFREE, an Energy Frontier Research Center 214 funded by the US Department of Energy (DE-SC-0001057; SAG). 
217 [1] P.A. Fleury, J.F. Scott, J.M. Worlock. Phys. Rev. Lett. 21 (1968) 16.

218 [2] H. Thomas, K.A. Müller. Phys. Rev. Lett. 21 (1968) 1256.

219 [3] K.A. Müller, H. Burkard, Phys. Rev. B 19 (1979) 3593.

220 [4] K. A. Müller, W. Berlinger, and E. Tosatti, Z. Phys. B 84, 277, 1991.

221 [5] R. Viana, P. Lunkenheimer, J. Hemberger, R. Böhmer, A. Loidl, Phys. Rev. B 50 (1994) 601.

222 [6] F. Schooley, W.R. Hosler, M.L.Cohen, Phys. Rev. Lett. 12 (1964) 474.

223 [7] T. Ishidate, T. Isonuma, Ferroelectrics 137 (1992) 45.

224 [8] M. Guennou, P. Bouvier, J. Kreisel Phys. Rev. B 81 (2010) 054115.

225 [9] W. Zhong, D. Vanderbilt, Phys. Rev. B 53 (1996) 5054.

226 [10] N. Sai, D. Vanderbilt, Phys. Rev. B 62 (2000) 13942.

227 [11] H. Salehi, J. Modern Physics. 2 (2011) 934.

228 [12] C.E. Ekuma, D. Bagayoko, M. Jarrell, J. Moreno, Material Sci. Cond. Matt. (2012) DOI $229 \quad 10.63 / 1.4751260$

230 [13] S. Piskunov, E. Heifets, R.I. Eglitis, G. Borstel, Computer Material Sci. 29 (2004) 165.

231 [14] E, Heifets, R.I. Eglitis, E.A. Kotomin, J. Maier, G. Borstel, Surface Sci. 513 (2002) 211.

232 [15] N.V. Krainyukova, V.V. Butskii, Surface Science 454-456(2000) 628.

233 [16] L. Rimai, G.A. deMars, Phys. Rev. 127 (1962) 702.

234 [17] S.A. Hayward, E.K.H. Salje, J. Phys. Chem. Solids 61(1999) 305.

235 [18] M.A. Carpenter, Amer. Mineral 92 (2007) 309.

236 [19] E.K.H. Salje, M. Guennou, P. Bouvier, M.A. Carpenter, J. Kreisel, J. Phys. Condens. Matter 23 (2011) 237275901.

238 [20] E.N. Maslen, N. Spadaccini, T. Ito,F. Marumo, Y. Satow, Acta Cryst B51 (1995) 939.

239 [21] Y.A. Abramov, V.G. Tsirelson, V.E.Zavodnik, S.A. Ivanov, I.D. Brown, Acta Cryst B51 (1995) 942.

240 [22] W. Jauch, M. Reehuis, Acta Cryst. A61 (2005) 411.

241 [23] R. Ranjan, D. Pandey, N.P. Lalla, Phys. Rev. Lett. 84 (2000) 3726.

242 [24] E.A. Zhurova, V.G. Tsirelson, Acta Cryst. B58 (2002) 567.

243 [25] V. Tsirelson, A. Stash, Acta Cryst. B58 (2002) 780.

244 [26] T. Yamanaka, T. Fukuda, T. Hattori, Rev. Sci. Instrum. 72 (2001) 1458. 
245 [27] H.K. Mao, J. Xu, P.M. Bell, J. Geophys. Res. 91 (1986) 4673.

246 [28] J. Friis, B. Jiang, J. Spence, K. Marthinsen, R. Holmestad, Acta Cryst. A60 (2004) 402.

247 [29] T.Yamanaka, T. Okada, Y. Nakamoto, Phys. Rev. B 80 (2009) 094108.

248 [30] P. Blaha, K. Schwarz, P. Dufek, G. Vielsack, W. Weber, Z. Naturforsch. 48a (1993) 129.

249 [31] R.E. Cohen, H. Krakauer, Phys. Rev. B 42 (1990) 6416.

250 [32] J. Hutton, R.J. Nelmes, J. Phys. C: Solid State Phys, 14 (1981) 1713.

251 [33] J.M. Kiat, G. Baldlinozzi, M. Dunlop, C. Malibent, B. Dkhil, C. Menoret, O. Masson, M. T. 252 Fernandez-Diaz, J. Phys. Cond. Matter, 12 (2000) 8411.

253 [34] T. Yamanaka, Y. Takeuchi, M. Tokonami, Acta Cryst. B40 (1984) 96.

254 [35] T. Yamanaka, M. Ahart, Y. Nakamoto, Z.G. Ye, S.A. Gramsch, H.K. Mao, R.J. Hemley, Phys. Rev. B $25586(2012) 174108$.

256 [36] R.D. King-Smith, D. Vanderbilt, Phys. Rev. B 49 (1994) 5828.

257 [37] S. Tinte, M.G. Stachiotti, Phys. Rev. B 58 (1998) 11959.

258 [38] F. Cora, C.R.A. Catlow, Faraday Discuss. 114 (1999) 421.

259 [39] M.J. Frisch, (2009) Gaussian 09 Gaussian, Inc., Wallingford, Connecticut. 
262 Fig. 1 Solid and open circles respectively indicate the lattice constants of $a$ and $c$ from the high-pressure and 263 low-temperature experiments. The lattice constants of $a_{t}{ }^{*}$ and $c_{t}{ }^{*}$ of the tetragonal phase are normalized to the 264 pseudocubic lattice $\left(a_{t}{ }^{*}=1 / \sqrt{2} a_{\mathrm{tetr}}\right.$ and $\left.c_{t}{ }^{*}=1 / 2 c_{\mathrm{tetr}}\right)$. The error in the lattice constant is less than the presented 265 circles and squares.

266 Fig. 2 Residual electron density distribution in the D-F maps on the (001) plane of the $\mathrm{SrTiO}_{3}$ cubic phase at 267 ambient conditions shows the $d-p$ - $\pi$ hybridization between $t_{2 g}$ electron of $\mathrm{Ti}(3 d)$ and $p_{x}$ electron of $\mathrm{O}(2 p)$. $\mathrm{TiO}_{6}$ 268 octahedron in the cubic phase has the site symmetry of $m 3 m$. Dotted lines show the $d-p-\pi$ hybridization.

269 Fig. 3 Harmonic oscillation model (left side) and anharmonic model (right side) in the D-F map on (001) 270 plane show the ED distributions at the $\mathrm{TiO}_{6}$ and $\mathrm{SrO}_{8}$ polyhedron at ambient conditions. Four positive peaks 271 near Ti and $\mathrm{Sr}$ atoms in the cubic phase found in harmonic model disappear in consideration of anharmonic 272 thermal vibration atoms.

273 Fig. 4 MO calculation is executed using the method of HF and basis set of 3-21G. In the MO on the 274 projection perpendicular to the $<010>$ direction, Red and green colors are positive and negative orbitals, 275 respectively. Hybridization between $\mathrm{Ti}(3 d)$ and $\mathrm{O}(2 p)$ at the $\mathrm{TiO}_{6}$ octahedral site forms a $d-p$ - $\pi$ bond. Obvious 276 hybridization is not found around $\mathrm{Sr}$ atom.

\section{Table caption}

279 Table 1 Bond distance

280 The numbers in parentheses denote errors of the last dismal. Tolerant factors are obtained from the observable 281 average bond distances.

282 Table 2 Mulliken charges

283 Mulliken charges of all atoms in STO are individually determined by using the model of HF/3-21G. The 284 calculated charge of $\mathrm{O}_{\text {cal }}$ is defined for the neutrality of the bulk crystal. Those of O1(-2e) and O2(-2e) are

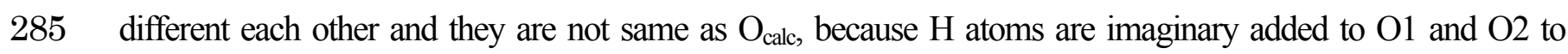
286 neglect the lone-paired coordination. 
Table 1

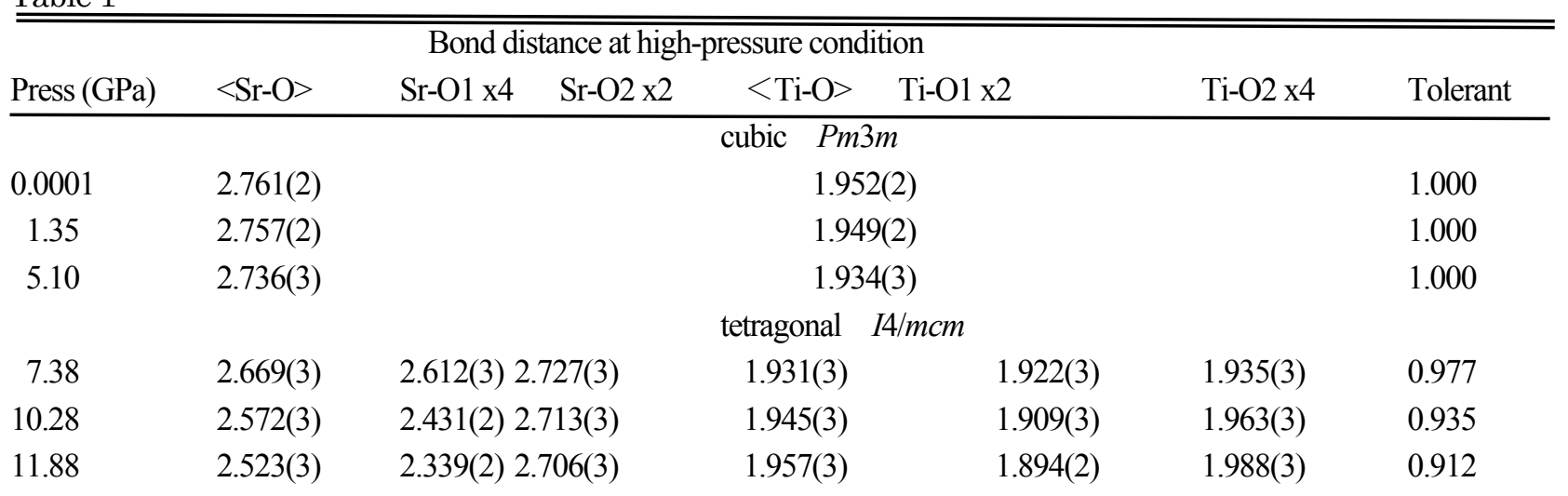

\begin{tabular}{|c|c|c|c|c|c|c|c|}
\hline \multirow[b]{2}{*}{ Temp (K) } & \multicolumn{5}{|c|}{ Bond distance at low temperature } & \multirow[b]{2}{*}{ Ti-O2 $x 4$} & \multirow[b]{2}{*}{ Tolerant } \\
\hline & $<\mathrm{Sr}-\mathrm{O}>$ & $\mathrm{Sr}-\mathrm{O} 1 \times 4$ & $\mathrm{Sr}-\mathrm{O} 2 \times 2$ & $<\mathrm{Ti}-\mathrm{O}>$ & Ti-O1 x2 & & \\
\hline \multicolumn{8}{|c|}{ cubic $\quad P m 3 m$} \\
\hline 300 & $2.761(5)$ & \multicolumn{5}{|c|}{$1.952(5)$} & 1.000 \\
\hline 190 & $2.761(5)$ & \multicolumn{5}{|c|}{$1.952(5)$} & 1.000 \\
\hline 120 & $2.760(6)$ & \multicolumn{5}{|c|}{$1.951(6)$} & 1.000 \\
\hline \multirow[t]{2}{*}{100} & $2.758(6)$ & \multicolumn{5}{|c|}{$\begin{array}{l}1.960(6) \\
\end{array}$} & 1.000 \\
\hline & & & & tetragonal & $I 4 / \mathrm{mcm}$ & & \\
\hline 95 & $2.630(6)$ & $2.759(6) 2$ & $500(7)$ & $1.977(6)$ & $1.952(6)$ & $1.989(5)$ & 0.941 \\
\hline 85 & $2.603(6)$ & $2.755(6) 2$ & $451(5)$ & $1.986(6)$ & $1.988(6)$ & $2.003(5)$ & 0.927 \\
\hline
\end{tabular}


Table 2

Bond distance and Mulliken population change at high pressure

\begin{tabular}{|c|c|c|c|c|c|}
\hline & \multicolumn{5}{|c|}{ cubic $\quad P m 3 m$} \\
\hline $\mathrm{P}(\mathrm{GPa})$ & $\operatorname{Sr}(2 \mathrm{e})$ & $\operatorname{Ti}(4 \mathrm{e})$ & $\mathrm{O}(-2 \mathrm{e})$ & & $\mathrm{O}_{\text {calc }}$ \\
\hline 0.0001 & 1.174 & 1.786 & -0.883 & & -0.987 \\
\hline 1.35 & 1.032 & 1.874 & -0.850 & & -0.968 \\
\hline \multirow[t]{3}{*}{5.10} & 0.949 & 1.883 & -0.894 & & -0.944 \\
\hline & \multicolumn{5}{|c|}{ tetragonal $\quad 14 / \mathrm{mcm}$} \\
\hline & $\operatorname{Sr}(2 \mathrm{e})$ & Ti4e1) & $\mathrm{O} 1(-2 \mathrm{e})$ & $\mathrm{O} 2(-2 \mathrm{e})$ & $\mathrm{O}_{\text {calc }}$ \\
\hline 7.38 & 1.133 & 1.929 & -0.934 & -0.906 & -1.021 \\
\hline 10.28 & 1.141 & 1.948 & -0.936 & -0.914 & -1.030 \\
\hline 11.88 & 1.227 & 1.989 & -0.935 & -0.921 & -1.072 \\
\hline
\end{tabular}

Bond distance and Mulliken population change at low temperature

\begin{tabular}{|c|c|c|c|c|c|}
\hline & \multicolumn{5}{|c|}{ cubic $\quad P m 3 m$} \\
\hline $\mathrm{T}(\mathrm{K})$ & $\operatorname{Sr}(2 \mathrm{e})$ & Ti(4e) & $\mathrm{O}(-2 \mathrm{e})$ & & $\mathrm{O}_{\text {calc }}$ \\
\hline 300 & 1.174 & 1.786 & -0.883 & & -0.987 \\
\hline 190 & 0.925 & 1.830 & -0.890 & & -0.918 \\
\hline 120 & 0.865 & 1.834 & -0.895 & & -0.899 \\
\hline \multirow[t]{3}{*}{100} & 0.864 & 1.834 & -0.896 & & -0.899 \\
\hline & \multicolumn{5}{|c|}{ tetragonal $\quad I 4 / \mathrm{mcm}$} \\
\hline & $\operatorname{Sr}(2 \mathrm{e})$ & $\operatorname{Ti}(4 \mathrm{e})$ & $\mathrm{O} 1(-2 \mathrm{e})$ & $\mathrm{O} 2(-2 \mathrm{e})$ & $\mathrm{O}_{\text {calc }}$ \\
\hline 95 & 1.131 & 1.886 & -0.891 & -0.904 & -1.005 \\
\hline 80 & 1.177 & 1.850 & -0.894 & -0.907 & -1.009 \\
\hline
\end{tabular}




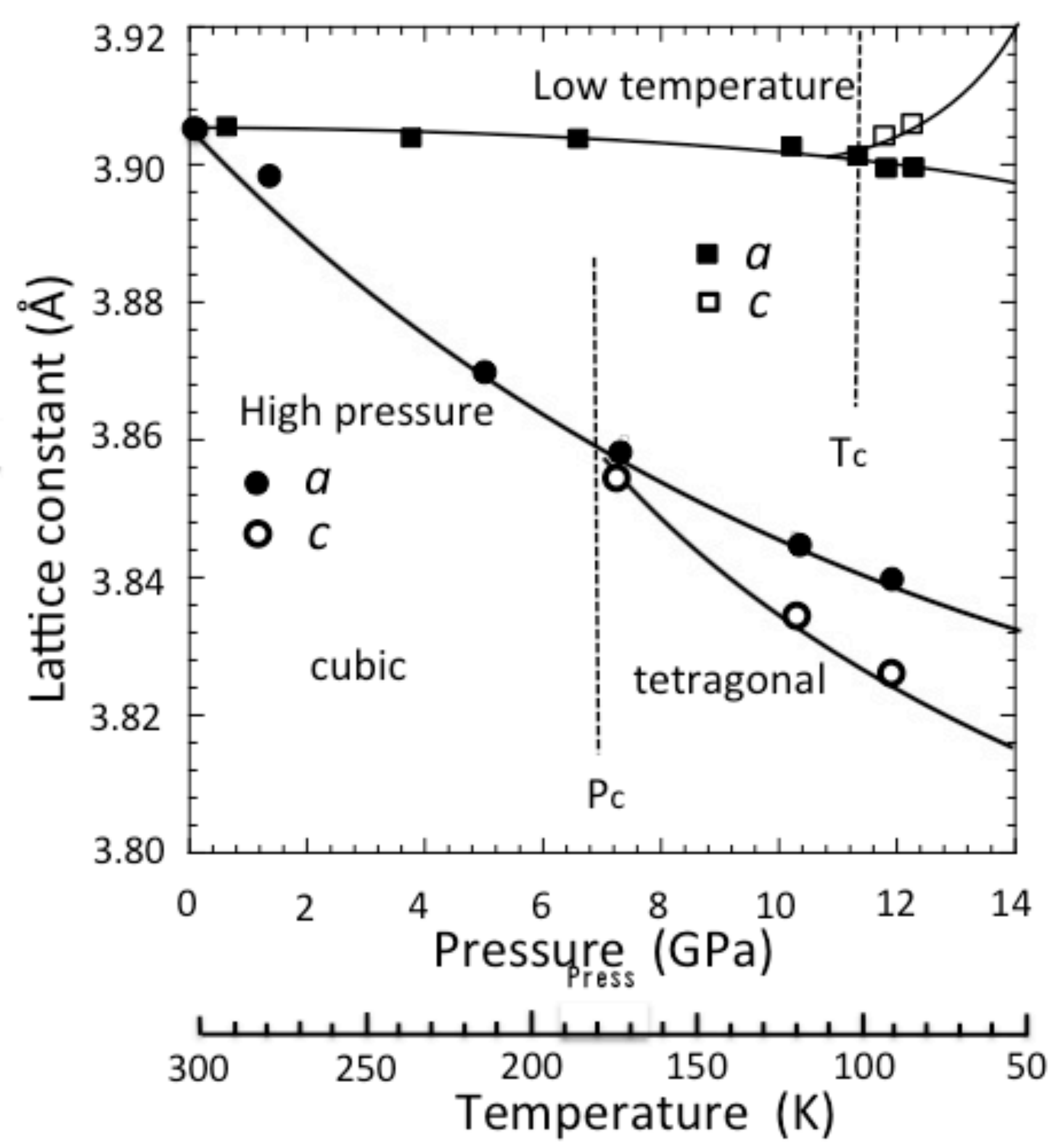




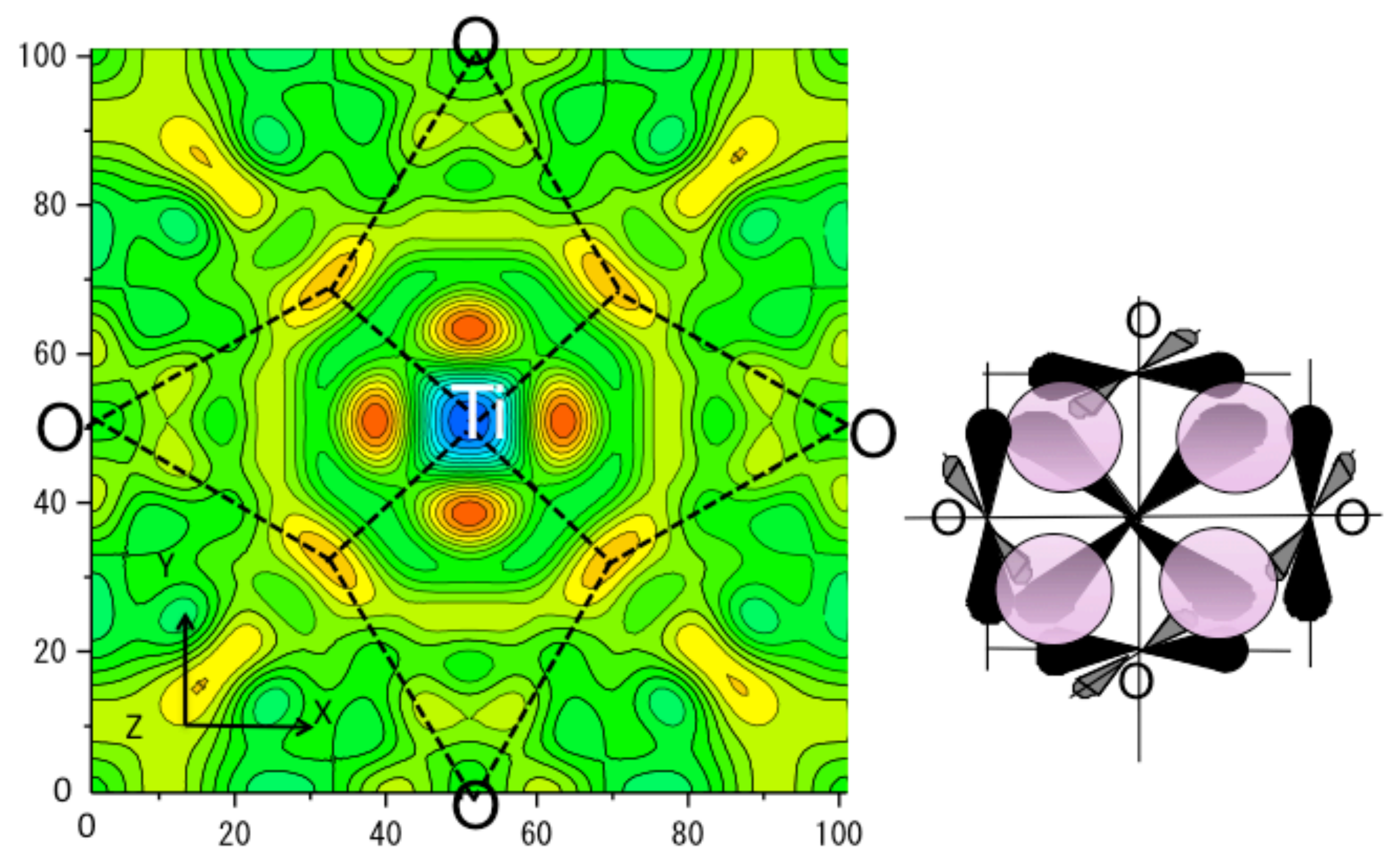


Harmonic oscillator model
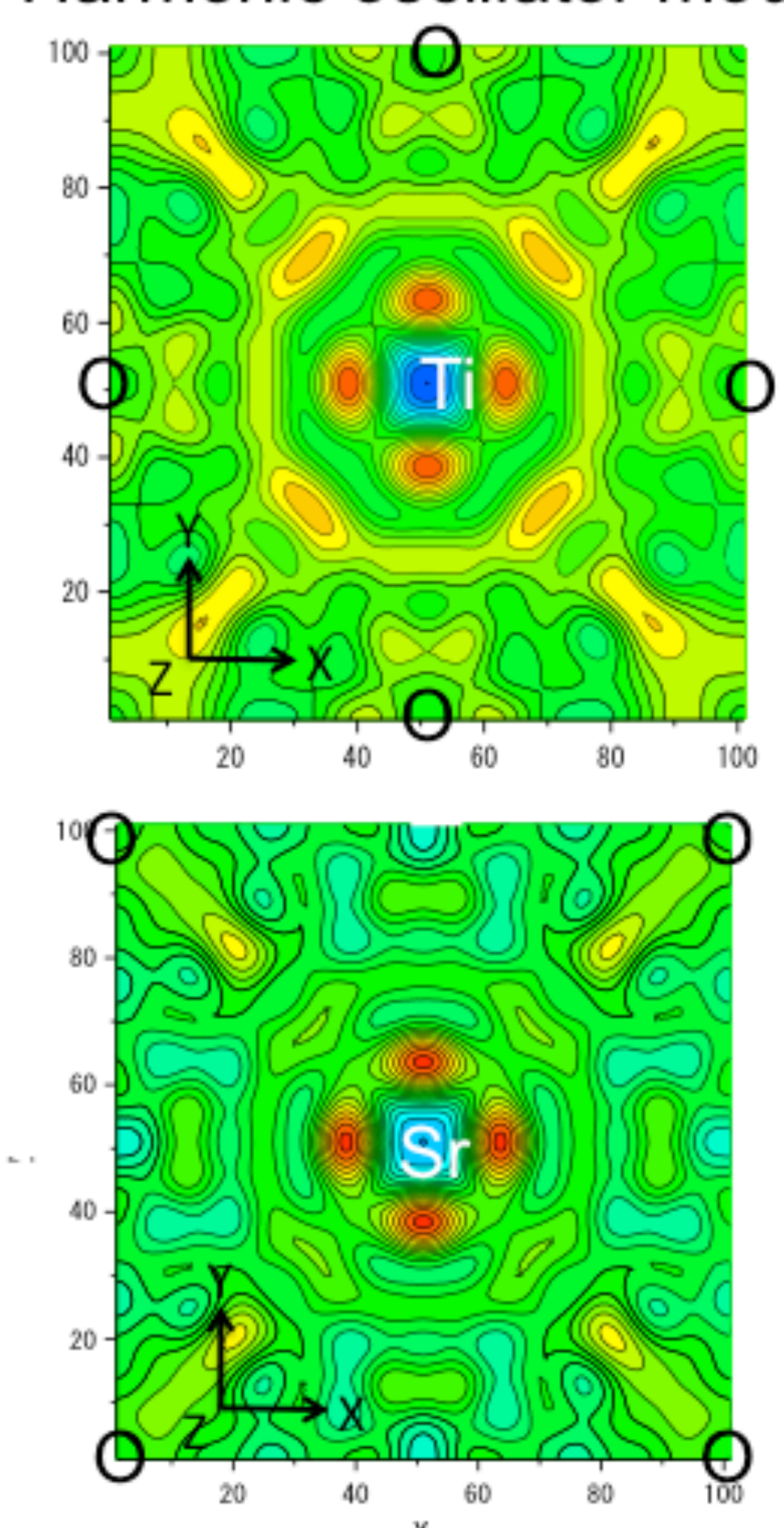

Anharmonic oscillator model
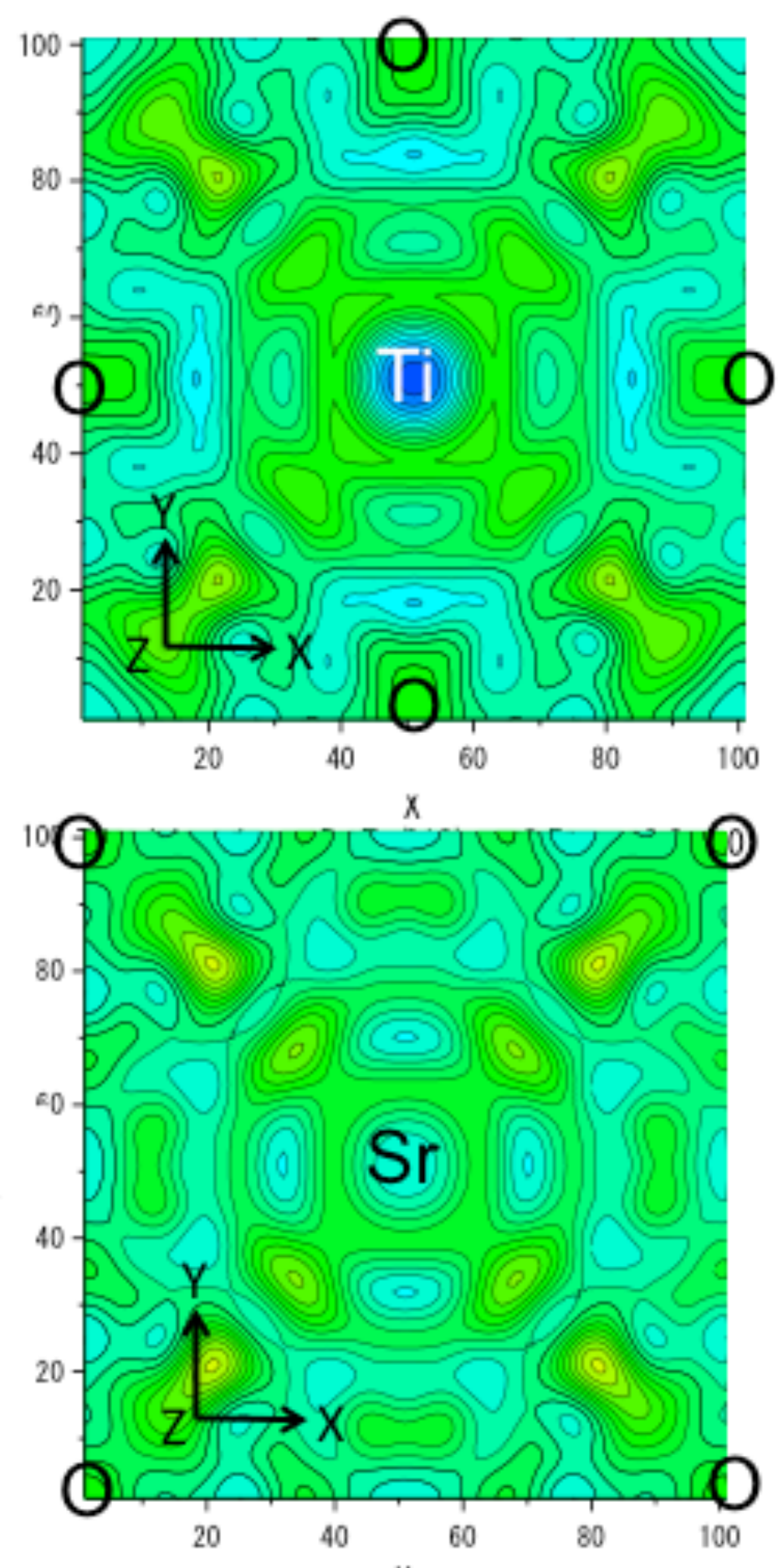

Fig. 3 
